\title{
Transformación y tendencias de la agricultura de Nueva Zelanda
}

\author{
José Jaime López Jiménez*
}

Introducción

$\mathrm{E}$ ste trabajo tiene como propósito principal explicar el proceso de transformación de la agricultura de Nueva Zelanda, resaltando las principales características de este proceso e identificando los factores más relevantes que han desempeñado un papel fundamental en el desarrollo del sector. Lo que se pretende es destacar los factores externos, locales y particulares que han caracterizado a la experiencia de la agricultura de Nueva Zelanda, desde su temprana vinculación con el mercado internacional hasta las tendencias más recientes.

El trabajo inicia con una breve descripción físico-geográfica de Nueva Zelanda. Después se expone cómo se fue configurando el esquema proteccionista mediante el cual el gobierno ejerció una fuerte intervención en el sector agropecuario, particularmente desde principios de los años sesenta hasta inicios de los ochenta. Posteriormente se explica el proceso de liberalización del sector agropecuario, presentándose los principales cambios introducidos en la política agrícola y la nueva visión del gobierno respecto a su función en el desarrollo del sector. En este sentido, una de las cuestiones que más han

* Profesor-investigador del Departamento de Estudios del Pacífico, de la Universidad de Guadalajara, e investigador del sNI. llamado la atención de la experiencia de Nueva Zelanda fue la rápida desregulación de su sector agropecuario, lo cual implicó significativos cambios en la estructura de producción y en la forma en que ahora se desenvuelven los productores en general, en un ambiente menos regulado por el Estado y más orientado hacia el mercado. Finalmente, se presentan las conclusiones del trabajo.

\section{Ubicación y descripción de Nueva Zelanda}

Nueva Zelanda es un pequeño país localizado en Oceanía, en la porción suroeste del Océano Pacífico, aproximadamente a 1,600 kilómetros al sureste de Australia. Es un territorio estrecho, alargado y recortado de aproximadamente 1,600 kilómetros que se extiende de norte a sur, con una anchura que, salvo algunas excepciones, no sobrepasa los 200 kilómetros. Cuenta con una superficie total de 270,534 kilómetros cuadrados - superficie similar a la de Japón o la de Gran Bretaña-; además posee 4,800 kilómetros de litoral tanto en el Océano Pacífico Sur como en el Mar de Tasmania. En sí, se trata de un archipiélago integrado por dos grandes islas (la Isla Norte y la Isla Sur), y un gran número de otras pequeñas. La Isla Norte, aunque es la de menor tamaño es la más poblada, ya que en ella se localizan los principales centros urbanos, como Auckland y Wellington, la capital del 
país. La Isla Sur es la de mayor extensión, la más accidentada y en donde se ubica la mayor proporción de los recursos naturales de que dispone Nueva Zelanda.

En general su superficie es predominantemente montañosa, con algunas planicies costeras extensas. Sólo una cuarta parte del territorio neozelandés se ubica por debajo de los 200 metros sobre el nivel del mar, por lo que las altas colinas y montañas nevadas dominan el paisaje, algunas de las cuales superan los 3,000 metros de altitud. Debido a su localización, su topografía y sus extensos litorales, Nueva Zelanda posee un clima marítimo templado, con abundantes precipitaciones que se distribuyen durante todo el año, mismas que van de los 380 milímetros en las áreas más secas del Este a los 8,000 milímetros en las áreas más húmedas del Oeste. ${ }^{1}$ Esta combinación de factores ha dotado a Nueva Zelanda de una densa vegetación de bosque subtropical y austral, ricas praderas y pastizales, que favorecen principalmente el desarrollo de las actividades forestales y de la ganadería.

Cabe subrayar que el recurso natural más importante de Nueva Zelanda es su tierra. De la superficie total del país, 6 por ciento se utiliza en la agricultura, 60 por ciento en las actividades pecuarias y 30 por ciento en actividades forestales. Pero también este país cuenta con ricos recursos energéticos, principalmente yacimientos de gas natural, y un considerable potencial hidroeléctrico apoyado en sus numerosos ríos de fuerte pendiente por lo accidentado del relieve, lo que favorece la generación de energía eléctrica limpia y barata para consumo doméstico y para las actividades económicas en general.

Actualmente su población asciende a casi cuatro millones de habitantes - pobla- ción similar a la de la zona metropolitana de Guadalajara-, de los cuales alrededor de 80 por ciento son de raza blanca — descendientes de inmigrantes británicos e irlandeses principalmente-, 15 por ciento son nativos maorí y el resto pertenece a grupos étnicos provenientes de India, China y de las Islas Polinesias del Pacífico. Aunque la economía es altamente dependiente del sector agropecuario, la mayor parte de la población, cerca de 85 por ciento, vive en áreas urbanas, predominantemente en la Isla Norte, en donde se ubica Auckland, la ciudad más grande del país - con un millón de habitantes-, incluso más grande que la capital Wellington. ${ }^{2}$

En el contexto internacional, Nueva Zelanda forma parte de diversos foros de cooperación regional como APEC y ASEAN, y del selecto grupo de países miembros de la Organización para la Cooperación y el Desarrollo Económico (ocDE). En 2004 alcanzó un producto interno bruto (PIB) de 98 mil millones de dólares americanos, ${ }^{3} \mathrm{y}$ un ingreso per cápita de 18,500 dólares internacionales —el cual representa más de dos veces el correspondiente para México-, ocupando el décimo-octavo lugar por este concepto entre los países desarrollados. ${ }^{4} \mathrm{De}$ igual forma, destaca en el comercio internacional por el elevado grado de apertura de su economía alcanzada después de las reformas económicas iniciadas en 1984. En 2005 sus exportaciones totales ascendieron a 22 mil millones de dólares, en tanto que sus importaciones alcanzaron cerca de 24 mil millones de dólares, en donde el sector agroalimentario participa con 53 por ciento del total -62 por ciento si se incluyen productos forestales-, y se ubica entre las 10 principales economías de mayor orientación al exterior a nivel mundial. ${ }^{5}$ 
Análisis

\section{El sector agropecuario de Nueva Zelanda}

\section{La protección del sector agropecuario hasta antes de 1984}

Desde fines del siglo XIX Nueva Zelanda tuvo una larga tradición de participación del gobierno en la agricultura, lo que propició una estrecha relación entre autoridades y agricultores, al grado de que estos últimos llegaron a acumular una fuerte influencia política en los principales círculos de poder y obtuvieron de la legislación y de las instituciones los apoyos que necesitaban. Fue así como a partir de 1922 se establecieron juntas de productores bien organizadas con facultades legales para controlar la producción y comercialización de la producción agropecuaria, entre las que destacaban las juntas de productores de la carne y de lana. ${ }^{6}$

A pesar de ello, hasta principios de los cincuenta el sector agropecuario de Nueva Zelanda recibió realmente un apoyo mínimo por parte del gobierno, en vista de que las ventajas comparativas en la agricultura eran tan fuertes que los agricultores requerían poca ayuda. Sin embargo, a finales de esa misma década los precios de los productos agropecuarios en los mercados internacionales tendieron a la baja, afectando significativamente a Nueva Zelanda al ser éstos la base de sus exportaciones. Esta situación se tradujo en un deterioro en el comercio exterior, provocando el surgimiento de un déficit en la balanza comercial del país.

Ante este panorama adverso se aceptó que la estrategia de sustitución de importaciones que se había adoptado a principios de los años treinta, por sí sola no podría proporcionar una solución a largo plazo para superar los problemas de la balanza de pagos y la falta de crecimiento de la economía, por lo que se consideró la necesidad de aumentar los ingresos vía la recuperación de las exportaciones agropecuarias. Fue así como durante los años sesenta el gobierno decidió establecer diversos programas de apoyo a la producción ganadera. El objetivo era simple: el aumento en la producción de carne de ovino y bovino, de lana y de productos lácteos principalmente, permitiría aumentar las exportaciones y esto, a su vez, permitiría financiar las importaciones, especialmente insumos para la modernización de la industria, que entonces alcanzaba ya altos niveles de protección.

Cabe aclarar que hasta principios de los sesenta la intervención del gobierno en la agricultura había estado limitada a la supervisión y apoyo a las organizaciones o juntas de productores, la mayoría de las cuales controlaban la producción y comercialización de los productos agropecuarios. Por lo tanto, la nueva asistencia del gobierno a la agricultura por medio de subsidios a la producción, que consistían en pagos directos al productor, significó un nuevo enfoque de la política agrícola a partir de 1963. Paralelamente, dentro de la nueva política de apoyo al sector agropecuario, especialmente a los ganaderos, se otorgaron subsidios a los fertilizantes y al crédito, concesiones sobre las tasas de interés y los impuestos e incentivos a las exportaciones, convirtiéndose todos ellos en importantes instrumentos de la política agrícola. Además, se proporcionaron apoyos para la investigación agropecuaria y los servicios de extensión.

Con el alza en los precios de los productos agrícolas a nivel internacional en los años setenta, los subsidios a los insumos fueron recortados internamente al considerarse que no eran ya tan nece- 
sarios. Pero, al no crecer la producción tan rápidamente como se esperaba, y con el posterior colapso de los precios internacionales, en general producto de la crisis del petróleo, el gobierno decidió otorgarle una mayor ayuda al sector agropecuario mediante nuevos esquemas de apoyo. Las nuevas medidas de apoyo se otorgaron principalmente mediante esquemas de precios mínimos suplementarios (SMP, por sus siglas en inglés) - equivalente a los precios de garantía establecidos en México a inicios de los sesenta-, con los cuales se buscó estimular la producción, proteger el ingreso de los agricultores y fortalecer las juntas de productores. Con ello, los precios de los principales productos agropecuarios quedaron regulados y la producción de los mismos quedó fuertemente protegida y controlada por los productores. ${ }^{7}$

A principios de los ochenta el sector agropecuario de Nueva Zelanda tenía una participación importante en la economía del país: el valor agregado de la producción agropecuaria representaba alrededor de 10 por ciento del PIB total, absorbía 11 por ciento de la población económicamente activa, y las exportaciones agropecuarias participaban con 66 por ciento del total.
Por su parte, las importaciones agropecuarias ascendían a cerca de 7 por ciento en promedio de las importaciones totales. ${ }^{8}$

Dentro del sector agropecuario destacaban las actividades pecuarias dado el mayor peso relativo del volumen y valor de su producción, sobresaliendo productos como la carne de ovino y bovino, leche fresca, leche en polvo, queso, mantequilla y lana. Sin embargo, con excepción de la leche en polvo, el crecimiento de los productos pecuarios en general - que en su mayor parte se destinan a la exportación-, paulatinamente fue disminuyendo y perdiendo importancia dentro del sector. Por el contrario, la producción de frutas y hortalizas experimentó un rápido crecimiento, al aumentar su producción en alrededor de 45 por ciento entre 1970 y 1980. Esto ofrece una idea del acelerado impulso que cobraron los cultivos agrícolas en los años previos a la reforma, debido a la menor demanda de productos pecuarios en el exterior y a la estrategia de diversificación de mercados y productos de exportación, adoptada tras la incorporación de Gran Bretaña a la Comunidad Europea en 1973 (cuadro 1).

Respecto al empleo en el sector agropecuario, es conveniente subrayar que en

\section{Cuadro 1}

Nueva Zelanda: evolución de la producción agropecuaria (Índices de producción, base $1980=100$ )

\begin{tabular}{lccrrrrrrr}
\hline Año & Cereales & Hortalizas & Frutas & Carne & Leche fresca & Leche en polvo & Queso & Mantequilla & Lana \\
\hline 1965 & 57 & 70 & 48 & 72 & 87 & 13 & 100 & 96 & 88 \\
1970 & 77 & 69 & 68 & 89 & 89 & 21 & 95 & 94 & 94 \\
1975 & 89 & 80 & 72 & 95 & 91 & 31 & 84 & 94 & 82 \\
1980 & 100 & 100 & 100 & 100 & 100 & 100 & 100 & 100 & 100 \\
1985 & 157 & 118 & 162 & 117 & 118 & 180 & 120 & 109 & 105 \\
1990 & 115 & 147 & 237 & 99 & 112 & 227 & 112 & 101 & 87 \\
1995 & 100 & 279 & 277 & 118 & 139 & 447 & 187 & 104 & 81 \\
2000 & 115 & 249 & 259 & 118 & 179 & 562 & 246 & 125 & 72 \\
\hline
\end{tabular}

Fuente: elaborado con base en el Banco de datos de la FAO, Roma, 2001. 
Nueva Zelanda éste incluye al subsector agrícola y al subsector de procesamiento e insumos. El primero incluye propiamente a las actividades agrícolas y pecuarias, en tanto que el segundo está integrado por industrias que reciben una parte sustancial de sus insumos de la agricultura y la ganadería, así como de aquellas que producen fertilizantes, pesticidas y maquinaria agrícola. En 1980 el empleo del sector en conjunto alcanzaba alrededor de 21 por ciento del total, 11 por ciento empleado en el subsector agrícola y 10 por ciento en la

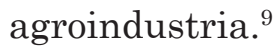

En el subsector agrícola el componente más grande del empleo está compuesto por mano de obra familiar, constituida por trabajadores y mano de obra familiar no asalariada, que en conjunto representaba alrededor de 80 por ciento del total del empleo en las actividades agrícolas y pecuarias a principios de los años ochenta. Pero también es importante mencionar que el uso de mano de obra familiar sin paga es elevado, ya que ésta absorbía una cuarta parte del total del empleo familiar y una quinta parte del total del empleo agrícola, situación que es poco común en los mercados de trabajo no agrícolas. El empleo pagado, por su parte, tanto de tiempo completo como parcial y temporal, representaba 25 por ciento del empleo agrícola total (cuadro 2).

De igual forma, el empleo femenino en las actividades agrícolas cumple un papel importante, aunque éste constituye en mayor medida mano de obra familiar sin paga y es básicamente de tiempo parcial. No obstante, la participación del trabajo femenino en el empleo total del sector aumentó paulatinamente durante las décadas de los setenta y ochenta, lo que ha llevado a más mujeres del medio rural a buscar empleo pagado en las actividades hortofrutícolas,
Cuadro 2

Nueva Zelanda: empleo en el sector agropecuario, 1980-1989

(porcentajes)

\begin{tabular}{lcc}
\hline Tipo de empleo & $1980-1984$ & $1985-1989$ \\
\hline Trabajadores propietarios & 56 & 55 \\
$\quad$ De tiempo completo & 71 & 72 \\
De tiempo parcial & 29 & 28 \\
Permanente pagado & 19 & 17 \\
$\quad$ De tiempo completo & 76 & 76 \\
De tiempo parcial & 24 & 24 \\
Temporal pagado & 6 & 7 \\
Subtotal & 81 & 79 \\
Familiar sin paga & 19 & 21 \\
Total & 100 & 100 \\
\hline
\end{tabular}

Fuente: OECD, Farm Employment and Economic

Adjustment in OECD Countries, Francia, 1994.

así como en el subsector de procesamiento y de servicios agropecuarios. La mejor infraestructura y los transportes, el crecimiento en el procesamiento de productos del campo y la mayor tecnificación de las actividades agropecuarias en general, han facilitado la creciente incorporación de la mujer en el empleo agropecuario pagado. ${ }^{10}$

\section{Los apoyos del gobierno al sector agropecuario}

Las transferencias totales del sector público destinadas al sector agropecuario de Nueva Zelanda, como resultado de la aplicación de la política agrícola proteccionista, llegaron a representar casi una quinta parte del valor total de la producción agropecuaria a principios de los ochenta; con ello la intervención del gobierno en el sector agropecuario alcanzó sus niveles más altos. En el periodo 1979-1981 el estimado del subsidio al productor (ESP) alcanzó un promedio anual de 18 por ciento, el cual aún se ubicaba muy por abajo en compa- 
Cuadro 3

\section{Equivalente del Subsidio al Productor por países (ESP)} (porcentajes)

\begin{tabular}{lccccc}
\hline País & $1979-1981$ & $1986-1988$ & $1991-1993$ & $1996-1998$ & $1998-2000$ \\
\hline Suiza & 64 & 74 & 71 & 69 & 71 \\
Noruega & 70 & 67 & 69 & 66 & 66 \\
Islandia & 68 & 75 & 71 & 60 & 66 \\
Corea del Sur & - & 71 & 76 & 65 & 66 \\
Japón & 60 & 65 & 58 & 63 & 63 \\
Unión Europea & 36 & 46 & 47 & 39 & 40 \\
Estados Unidos & 14 & 26 & 19 & 17 & 23 \\
Canadá & 20 & 34 & 30 & 15 & 18 \\
México & - & 10 & 34 & 14 & 16 \\
Australia & 8 & 7 & 8 & 6 & 6 \\
Nueva Zelanda & 18 & 11 & 2 & 1 & 1 \\
Promedio OCDE & 29 & 41 & 39 & 33 & 35 \\
\hline
\end{tabular}

Fuente: OECD, Agricultural Policies in OECD Countries. Monitoring and Evaluation, Francia, 1996,1999 y 2001.

ración con los países con el mayor nivel de subsidios al productor a nivel mundial (cuadro 3). Sin embargo, entre 1980 y 1984 el EsP se duplicó al pasar de 15 a 34 por ciento, lo que convirtió al país en una de las economías con el mayor nivel de subsidios agrícolas a escala mundial. ${ }^{11}$

De igual forma, los subsidios totales a la agricultura neozelandesa incrementaron su proporción respecto al PIB, al pasar de 2.0 a 3.1 por ciento en los primeros años de los ochenta. Este aumento en los subsidios del gobierno a la agricultura, también se vería reflejado en un mayor déficit público durante esos años. ${ }^{12} \mathrm{El}$ EsP por agricultor de tiempo completo en Nueva Zelanda alcanzó los 5,000 dólares americanos entre 1979 y 1981, cantidad similar a la otorgada por países como Estados Unidos, Canadá, Japón o la Unión Europea, pero bastante inferior a los 15,000 dólares en promedio por agricultor que otorgaban países como Noruega, Suecia y Suiza. ${ }^{13}$

En cuanto a la distribución de los apoyos dentro del sector agropecuario, cabe resaltar que éstos se centraban fundamentalmente en las actividades pecuarias, al ser éstas las de mayor peso. Entre los productos pecuarios con mayores subsidios destacaban la carne de ave, huevo, carne de porcino, carne de ovino, la leche y la lana. Del total de los subsidios, 72 por ciento se destinaba como apoyo a los precios, 8 por ciento a la tasa interés, 4 por ciento en concesiones de impuestos, 3 por ciento para la investigación y extensión, 3 por ciento para actividades de inspección y vigilancia, y 2 por ciento en fertilizantes. ${ }^{14}$ Lo que da una idea de las fuertes distorsiones en los precios agrícolas que prevalecían en esos años.

En 1983, finalmente el gobierno aceptó que el costo fiscal de los apoyos otorgados para estimular la producción agropecuaria durante las últimas dos décadas era insostenible, ante la disminución de los precios mundiales de algunos productos agrícolas y el creciente desequilibrio de las finanzas internas. Por otra parte, a pesar de los apoyos recibidos la situación de los exportadores neozelandeses había empeorado como resultado de las condiciones desfavorables de los mercados internacionales y de las 
políticas proteccionistas adoptadas por sus principales socios comerciales. ${ }^{15}$ De hecho, los apoyos otorgados al sector agropecuario habían sido insuficientes para estimular el crecimiento de las exportaciones agropecuarias, especialmente de los productos pecuarios que constituían la base del sector primario de Nueva Zelanda.

\section{La reestructuración del sector agropecuario de Nueva Zelanda}

\section{El retiro del Estado en la agricultura}

Como parte de las reformas económicas implementadas en $1984^{16}$ se anunció un amplio y profundo programa de liberalización del sector agropecuario, que incluía el recorte de los subsidios a los fertilizantes y al crédito, la eliminación de los controles sobre los precios agrícolas -el esquema de precios mínimos suplementarios-, la desaparición de las concesiones especiales sobre la tasa de interés y los impuestos de que habían disfrutado los agricultores y la eliminación de los incentivos a las exportaciones. Además, se privatizaron las empresas estatales productoras de insumos y se redujeron los apoyos a la investigación y a los servicios de extensión. La justificación era que el costo fiscal de las transferencias al sector agropecuario para mantener la producción, así como para enfrentar los declinantes términos de intercambio y los altos costos de los insumos provocados por la regulación y la protección manufacturera, se había vuelto insostenible para el gobierno.

Cabe señalar que la desregulación del sector agropecuario se inició en 1984, se intensificó en 1985 y fue completada en 1988; no obstante, el periodo de ajuste del sector, y de la economía en conjunto, se prolongó hasta 1992. Sobre este aspecto, vale la pena subrayar que se consideró que la reforma económica en general, y la liberalización de la agricultura en particular, deberían aplicarse rápidamente para reducir los costos del ajuste, evitar el reagrupamiento de grupos de interés para inhibir los cambios y no dar tiempo a los políticos de retractarse respecto a las reformas. ${ }^{17}$ Los mayores cambios consistieron en la eliminación de los esquemas de precios mínimos suplementarios y de los esquemas de crédito subsidiado para las juntas de productores. Con ello, la proporción del ESP disminuyó a 11 por ciento a fines de 1988, para ubicarse finalmente en 1 por ciento desde $1998 .{ }^{18}$

Sin embargo, la liberalización de la agricultura no implicó un retiro total por parte del Estado, ni se dejó todo en manos del mercado. En 1986 el gobierno decidió introducir esquemas de apoyo para facilitar el proceso de ajuste, destinados especialmente a aquellas unidades agrícolas marginales y poco viables que quedaron desprotegidas con la desregulación del sector. Entre las medidas de apoyo destacó un programa especial de ayuda a agricultores, que operó en el periodo 1986-1989, mediante el cual los productores recibieron subsidios directos para compensar las pérdidas en el ingreso rural ocasionadas por el recorte de los apoyos, algo similar a lo ocurrido en México con Procampo (gráfica 1).

Otra de las medidas de apoyo que se llevaron a cabo en el periodo de aplicación de la reforma, fue un esquema de descuentos de deuda por conducto del Banco Rural de Nueva Zelanda, lo que le permitió a muchos granjeros reestructurar o incluso cancelar su deuda. Como resultado, alrededor de 20 por ciento del total de la deuda del sector quedaría cancelada. ${ }^{19} \mathrm{Si}$ bien es cierto que con estas medidas el go- 


\section{Gráfica 1}

Nueva Zelanda: evolución del ESP por periodos

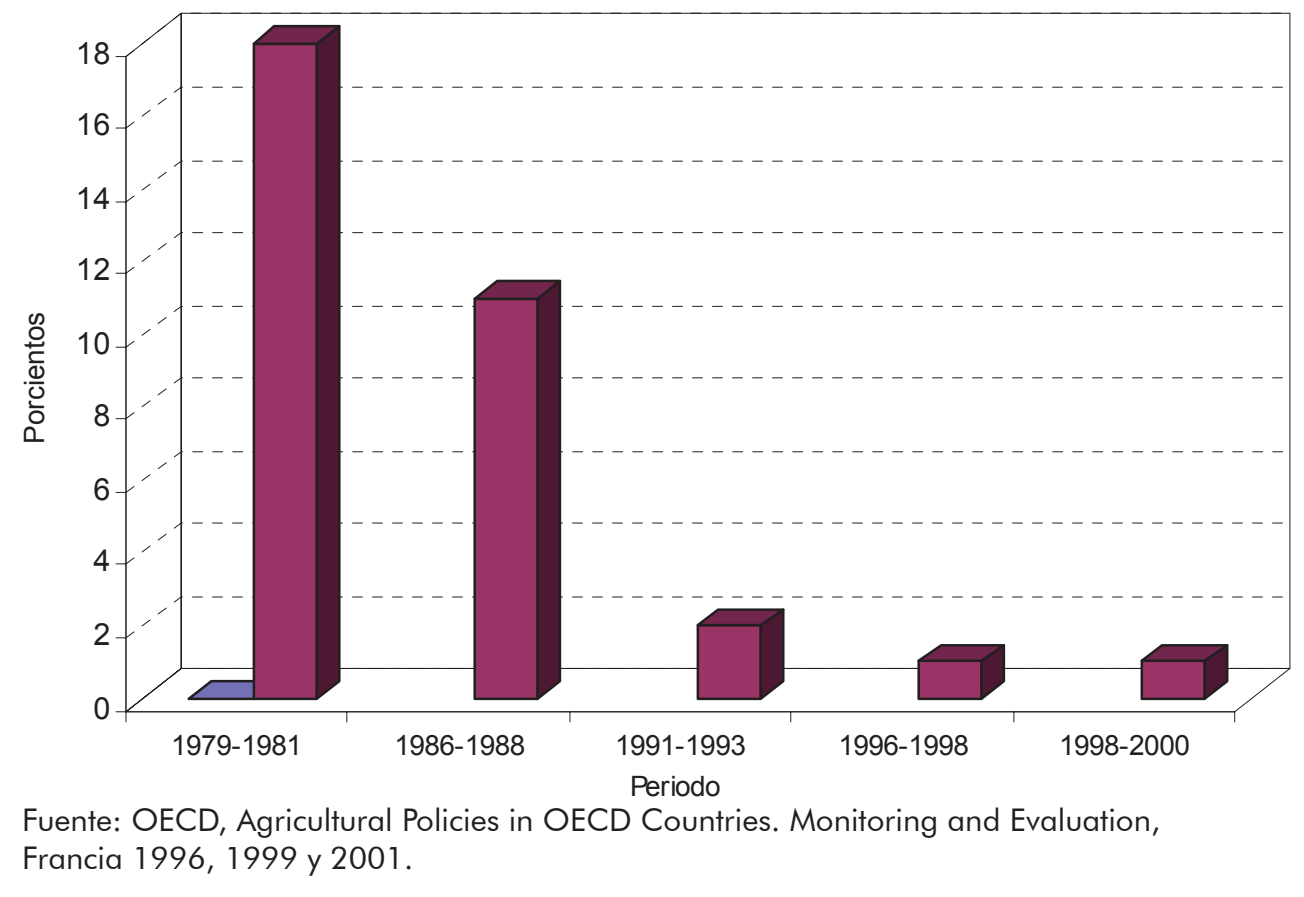

bierno no dejaba totalmente desprotegido al sector agropecuario, sobre todo a los productores de menores ingresos, para la gran mayoría de los productores y consumidores el rápido recorte de los subsidios fue una medida que afectó directamente su economía familiar.

\section{Principales cambios en el sector agropecuario en el corto plazo}

La liberalización del sector agropecuario iniciada en 1984 tuvo un efecto inmediato en la estructura de producción y en el ingreso de los productores, debido a que el recorte de los apoyos del gobierno y la desregulación del sector se dieron en forma mucho más rápida que en otros sectores económicos. Tras el repentino recorte de los apoyos durante los primeros años de la reforma, la inversión agropecuaria descendió en términos reales a sólo 30 por ciento del nivel alcanzado a principios de los ochenta. De igual forma, el ingreso agrícola disminuyó notablemente, al representar 85 por ciento del nivel promedio alcanzado a inicios de los años ochenta. ${ }^{20}$ Como resultado de estas condiciones desfavorables, la producción agrícola se estancó y su participación descendió de 7 a 6 por ciento del piB total de Nueva Zelanda entre 1984 y 1987 (gráfica 2)

Por otra parte, el valor de la tierra, que había alcanzado su máximo nivel a principios de los ochenta, cayó alrededor de 50 por ciento en términos reales, junto con el ingreso agrícola por las elevadas tasas de interés y el aumento en los costos de los insumos, los cuales implicaban mayores costos de operación. Esto dio lugar a que una gran cantidad de propiedades agrícolas perdieran su rentabilidad, estimándose que alrededor de 6,000 unidades de producción rural -las cuales representaban poco 


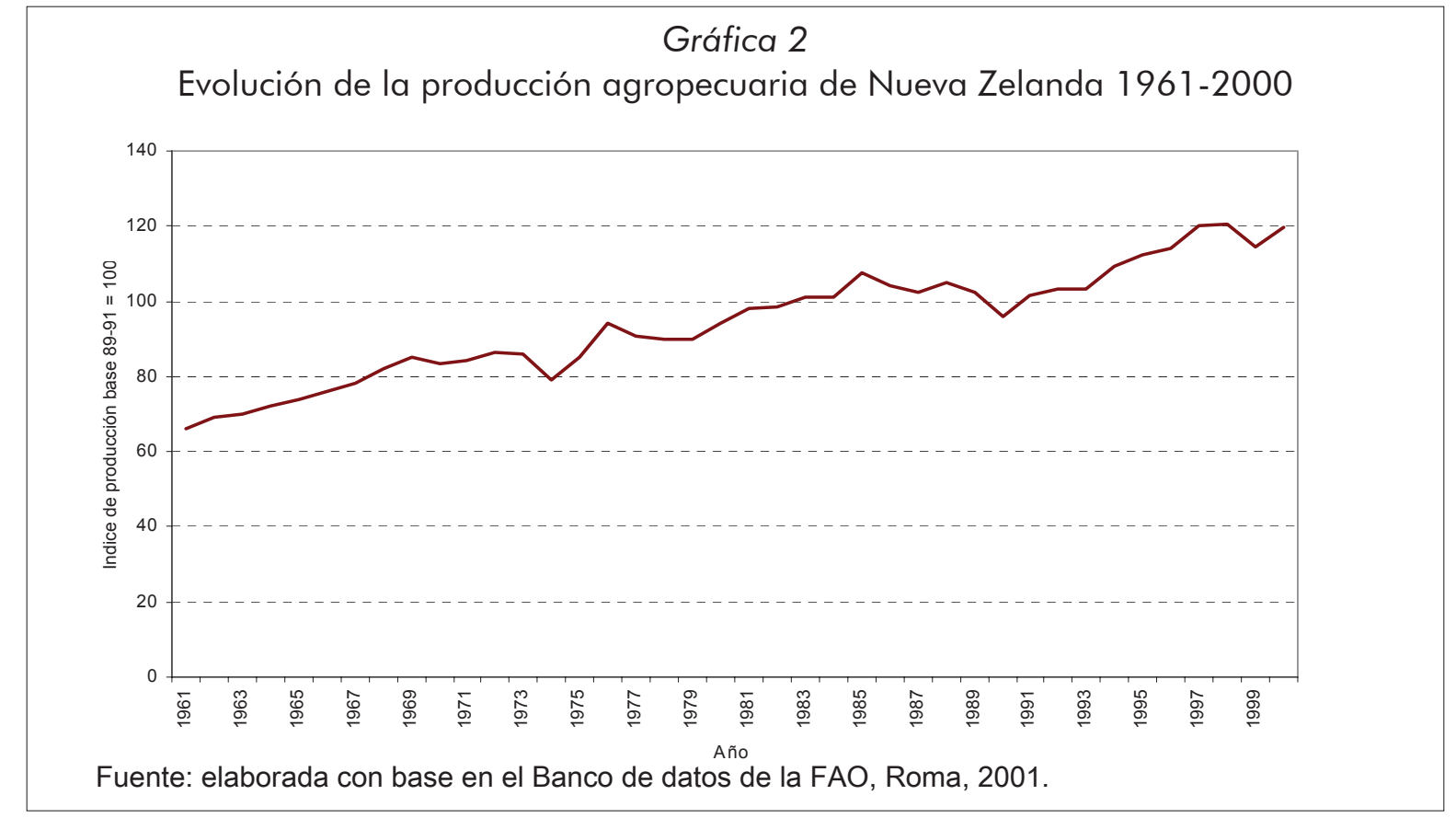

más de 8 por ciento del total-pasaron por una severa crisis financiera a finales de los ochenta. $^{21}$

De hecho, todos los subsectores agropecuarios fueron afectados, pero fueron los de carne de ovino y bovino los que sufrieron los cambios más drásticos y experimentaron los mayores ajustes. En términos absolutos, el ingreso agrícola bruto para estos últimos fue menor en 1.1 mil millones de dólares neozelandeses en promedio anual para el periodo 1984-1991, que en la década de los años setenta. ${ }^{22} \mathrm{Al}$ mismo tiempo, la eliminación del apoyo a los precios agrícolas y de los subsidios a los insumos agropecuarios, en combinación con la depreciación de los ingresos agrícolas y la devaluación de la moneda, propiciaron una menor demanda de insumos agropecuarios. La respuesta inicial de los agricultores fue reducir el gasto, especialmente en fertilizantes, mantenimiento y adquisición de maquinaria, lo que dio como resultado que las ventas de fertilizantes disminuyeron a la mitad de su volumen, al igual que la adquisición de equipo entre 1985 y $1988 .^{23}$

Esta reducción en el uso de insumos agropecuarios afectó significativamente el crecimiento de la producción agrícola, por lo que un gran número de agricultores se vieron en la necesidad de vender una parte de sus bienes de capital en un esfuerzo por mantener la viabilidad de sus actividades productivas. Además de los efectos inmediatos de reducir el uso de insumos agrícolas y en muchos casos de subdividir sus propiedades, los agricultores aplicaron en forma creciente diversas medidas para mantener sus granjas, como la venta de equipo excedente y de parcelas subutilizadas.

Por otra parte, como resultado de los cambios en los precios y en los costos al eliminarse la ayuda agrícola, las decisiones de producción de los agricultores se vieron influidas por las señales de los precios internacionales, optando así por cultivos 
más rentables, con un menor uso de insumos e inversión en capital. Esto trajo como consecuencia importantes cambios en la estructura productiva de Nueva Zelanda, observándose que mientras por un lado la producción de frutas y hortalizas siguió expandiéndose, por otro continuó el descenso en la producción de carne de ovino y de lana, por la pérdida de rentabilidad de las granjas de ganado ovino.

El número de unidades agrícolas aumentó de 69 mil a 71 mil propiedades durante el periodo 1985-1988, lo que representó un aumento de 9 por ciento respecto a las existentes en 1981. Mientras tanto, la escala promedio de las unidades de producción rural continuó disminuyendo al pasar de 227 a 220 hectáreas, principalmente por el aumento en el número de granjas productoras de frutas y hortalizas. Es importante mencionar que estos cambios, tanto en el número como en el tamaño de las unidades de producción rural, se registraron en su mayor parte en las propiedades menores de 40 hectáreas, particularmente en el rango de entre $20 \mathrm{y}$ menos de 40 hectáreas de superficie. Esto significa que los productores de menor escala de producción optaron por deshacerse de parte de sus propiedades, bien para poder solucionar problemas financieros derivados de la caída en las exportaciones agropecuarias y del aumento en sus costos de producción, o bien para convertir sus propiedades en granjas hortofrutícolas de menor tamaño (cuadro 4).

En relación con el empleo en las actividades agropecuarias, entre 1980 y 1984 éste presentó un descenso en términos absolutos y, con ello, una menor participación en el empleo total, aunque al interior del sector este proceso no fue homogéneo. Por ejemplo, mientras el empleo en las actividades pecuarias experimentó un descenso,

\section{Cuadro 4}

Nueva Zelanda: unidades de producción rural y escala de producción

\begin{tabular}{cccc}
\hline Año & $\begin{array}{c}\text { Unidades de } \\
\text { producción } \\
\text { (miles) }\end{array}$ & $\begin{array}{c}\text { Superficie } \\
\text { agropecuaria } \\
\text { (1000 has.) }\end{array}$ & $\begin{array}{c}\text { Escala } \\
\text { promedio } \\
\text { (Has.) }\end{array}$ \\
\hline 1976 & 62 & 16,110 & 261 \\
1981 & 65 & 15,999 & 245 \\
1982 & 65 & 15,939 & 245 \\
1983 & 66 & 15,899 & 240 \\
1984 & 68 & 15,787 & 233 \\
1985 & 69 & 15,731 & 227 \\
1986 & 70 & 15,698 & 224 \\
1987 & 70 & 15,673 & 222 \\
1988 & 71 & 15,491 & 220 \\
1989 & 71 & 15,888 & 224 \\
1990 & 71 & 15,316 & 216 \\
1993 & 81 & 16,336 & 202 \\
1998 & 81 & 16,578 & 204 \\
2000 & 80 & 15,500 & 193 \\
\hline
\end{tabular}

Fuente: OECD, Farm Employment and Economic Adjustment in OECD Countries, Francia, 1994; Federated Farmers of New Zealand, "Life After Subsidies", marzo 2002, Nueva Zelanda.

en las actividades agrícolas y forestales hubo un crecimiento, lo que en parte tiene su explicación por el rápido crecimiento de los cultivos hortofrutícolas y de las actividades forestales, al ser más intensivas en mano de obra. Esto significa que el crecimiento de la producción agrícola se dio como resultado de la reorientación de las actividades pecuarias hacia las agrícolas y del aumento en la productividad de la mano de obra agrícola (cuadro 5). En cuanto al ingreso de los agricultores, éste exhibió una notable recuperación entre 1980 y 1982 , pero el aumento en los precios internos como consecuencia de la devaluación de la moneda neozelandesa que ocurrió en esos años, ocasionó una fuerte caída en 1983. A pesar de ello, en 1984 el 
Análisis

Cuadro 5

Nueva Zelanda: empleo agropecuario según actividad y tipo de empleo, 1980-1989

(miles)

\begin{tabular}{lrrrrrrrrr}
\hline Actividad & \multicolumn{3}{c}{ Propietarios } & \multicolumn{3}{c}{ Pagados permanentes } & \multicolumn{3}{c}{ Pagados temporales } \\
\hline & 1980 & 1984 & 1989 & 1980 & 1984 & 1989 & 1980 & 1984 & 1989 \\
\hline Ganadería & 74.6 & 72.6 & 68.8 & 24.9 & 19.2 & 17.0 & 5.6 & 5.0 & 3.7 \\
Lácteos & 26.0 & 26.7 & 26.0 & 5.7 & 4.7 & 5.0 & 0.7 & 0.9 & 0.6 \\
Ovinos & 31.5 & 30.8 & 25.7 & 13.3 & 10.5 & 7.5 & 3.8 & 3.2 & 2.0 \\
Bovinos & 9.5 & 9.0 & 8.9 & 1.5 & 1.4 & 1.2 & 0.4 & 0.4 & 0.3 \\
Cerdos & 0.8 & 0.7 & 0.8 & 0.4 & 0.3 & 0.3 & 0.0 & 0.0 & 0.0 \\
Venados & 0.2 & 0.5 & 1.8 & 0.0 & 0.1 & 0.3 & 0.0 & 0.0 & 0.1 \\
Equinos sementales & 0.6 & 0.8 & 1.7 & 0.2 & 0.4 & 0.8 & 0.0 & 0.0 & 0.1 \\
Aves de corral & 0.8 & 0.6 & 0.6 & 0.9 & 0.7 & 0.8 & 0.1 & 0.2 & 0.2 \\
Ganadería mixta & 5.2 & 3.5 & 3.3 & 2.9 & 1.1 & 1.1 & 0.6 & 0.3 & 0.4 \\
Agrícultura & 13.3 & 13.8 & 14.1 & 10.4 & 11.0 & 8.0 & 5.4 & 6.9 & 5.8 \\
Labranza & 2.2 & 2.5 & 1.5 & 0.5 & 0.7 & 0.3 & 0.8 & 0.3 & 0.1 \\
Horticultura & 6.2 & 8.9 & 11.5 & 3.0 & 5.9 & 6.5 & 3.2 & 6.0 & 5.4 \\
Plantaciones & 0.5 & 0.2 & 0.5 & 4.0 & 3.5 & 0.7 & 0.2 & 0.2 & 0.1 \\
Otros cultivos & 4.4 & 2.2 & 0.6 & 2.9 & 0.9 & 0.5 & 1.2 & 0.4 & 0.2 \\
Total & 88.0 & 87.0 & 83.9 & 35.4 & 30.1 & 25.2 & 11.1 & 11.8 & 9.4 \\
\hline
\end{tabular}

Fuente: OECD, Farm Employment and Economic Adjustment in OECD Countries, Francia, 1994.

ingreso volvió a recuperarse, aunque sin llegar al nivel alcanzado a principios de los años ochenta. ${ }^{24}$

En el sector externo, como producto de las sucesivas devaluaciones del tipo de cambio, las exportaciones agropecuarias en general observaron una lenta recuperación al presentar un crecimiento medio anual de 5 por ciento, mientras su participación en el total de las exportaciones disminuyó al pasar de 66 a 54 por ciento a principios de los noventa. Esto se debió a que, no obstante el crecimiento en la participación de los lácteos y los productos hortofrutícolas, los principales productos de exportación - carne y lana - siguieron perdiendo importancia. En cuanto a las importaciones agropecuarias, empujadas por la apertura comercial, manifestaron un sostenido aumento al crecer 8 por ciento promedio anual hasta principios de los noventa, al mismo tiempo que presentaron un ligero ascenso en su participación respecto al total de las importaciones del país. Entre los principales productos importados siguieron figurando los cereales, azúcar, café, cacao, bebidas y tabaco, lo cual significa que no hubo mayores cambios en las importaciones de productos agropecuarios con la liberalización del sector.

\section{Cambios en el sector agropecuario en el largo plazo}

El número de las unidades de producción rural de Nueva Zelanda siguió aumentando conforme se fue recuperando la economía desde principios de los noventa, lo que aunado a otras variables, en cierta forma reflejaba la gradual recuperación de la producción y productividad del sector. Entre 1990 y 2000 las explotaciones agrícolas pasaron de 71 mil a 80 mil unidades, lo que representó un crecimiento de casi 13 por ciento en una década, pero la escala de producción continuó reduciéndose, al des- 
cender de 216 a 193 hectáreas. Aún así, la escala actual de las unidades de producción rural de Nueva Zelanda les ha permitido a los agricultores adaptarse de mejor manera al proceso de reestructuración mediante un mejor aprovechamiento de los factores de producción (tierra, trabajo y capital), una especialización productiva en cultivos de exportación, un mejor acceso a los mercados de capital y una mayor integración a la agroindustria global.

La superficie agrícola, por su parte, después de experimentar una serie de altibajos durante la década de los ochenta mostró un notable descenso a principios de los noventa, provocado principalmente por la disminución en el cultivo de cereales. De 1990 al 2000 la superficie agrícola disminuyó de 17.4 a 16.6 millones de hectáreas. La explicación de este proceso de contracción en la superficie agrícola y de creación de un mayor número de unidades de producción rural de menor tamaño, radica en que actualmente en Nueva Zelanda ha cobrado un mayor impulso la formación de numero- sas corporaciones agrícolas empresariales de tamaño medio dedicadas a la exportación. Esto significa que, si anteriormente la ampliación de la frontera agrícola permitió a su vez un crecimiento en el volumen y valor de la producción agropecuaria, ahora el crecimiento de la producción se explica principalmente por los incrementos en la productividad y en los rendimientos agrícolas, como resultado de la utilización de más y mejores tecnologías. ${ }^{25}$

$\mathrm{Al}$ interior de la producción agrícola es importante observar los notorios cambios que experimentó el campo de Nueva Zelanda con la reestructuración del sector. En el caso de los cereales - principalmente trigo, cebada y maíz-, hubo una paulatina reducción tanto en la superficie sembrada como en la producción, al disminuir el volumen producido en una tercera parte entre 1984 y 1999. En cuanto al cultivo de frutas y hortalizas, las primeras incrementaron su producción en casi 100 por ciento y las segundas en 80 por ciento durante el mismo periodo (gráfica 3). Entre los cultivos

Gráfica 3

Cambios en la estructura de producción agrícola de Nueva Zelanda 1970-1999

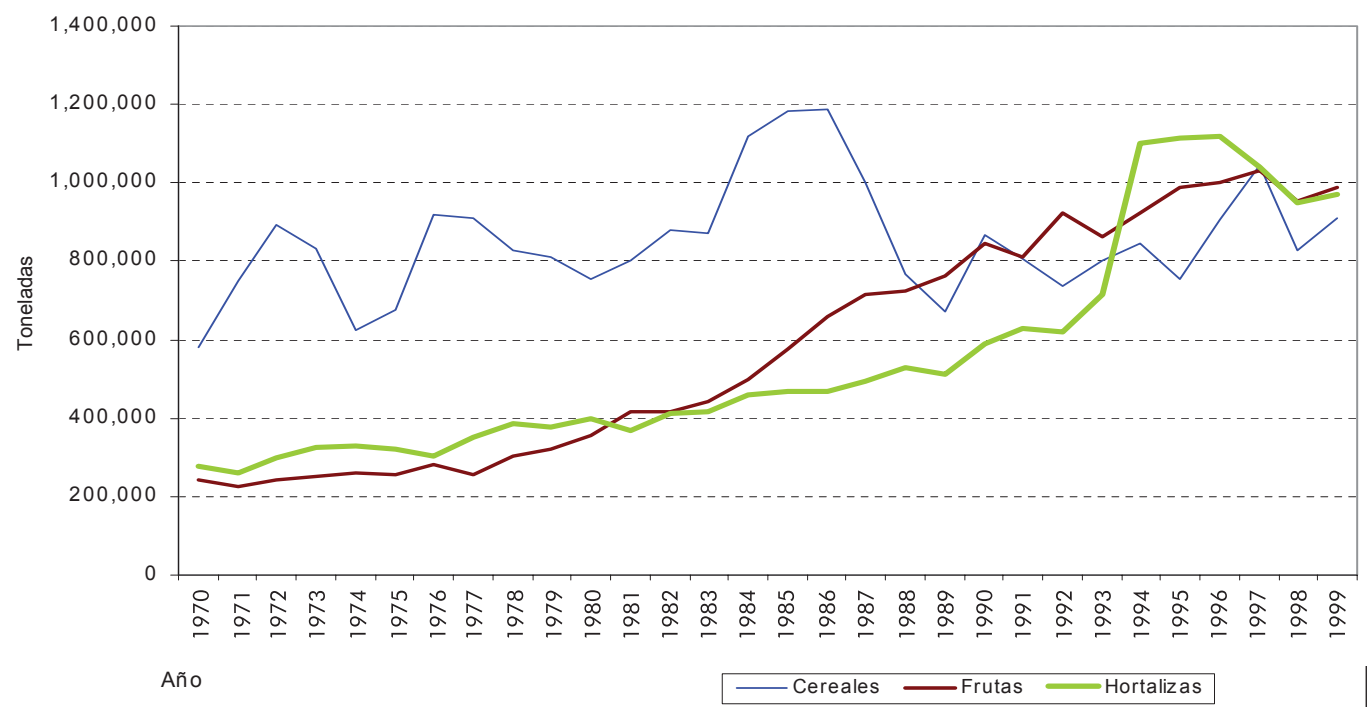

Fuente: elaborada con base en el Banco de datos de la FAO, Roma, 2001. 
agrícolas de mayor crecimiento sobresale la producción de kiwi, manzana y pera. ${ }^{26}$

El cambio más drástico ocurrido dentro de las actividades pecuarias fue el experimentado por el subsector ovinos, considerada como la actividad tradicional de este país. El inventario de ovinos se redujo de una población de más de 70 millones de ovejas en 1983, a menos de 45 millones en 1999, ante la pérdida de rentabilidad de esta actividad por la caída de los precios internacionales en la carne y lana de ovino, así como por las medidas de liberalización establecidas. A diferencia de lo ocurrido en el subsector de ovinos, se observó una tendencia creciente en el inventario de bovinos y aves en los años posteriores a la reestructuración del sector, lo que sugiere que los granjeros de Nueva Zelanda optaron por sustituir la producción de carne de ovino por la de bovino y de aves, e incluso por carne de venado (gráfica 4).

Con respecto a la PEA agrícola, ésta ha ido aumentando paulatinamente conforme se ha recuperado la producción agropecuaria. Entre 1993 y 1999 la fuerza de trabajo empleada en este sector pasó de 132 mil a 215 mil personas, aumentando también su participación de 8.5 a 11.4 por ciento de la fuerza de trabajo total. ${ }^{27}$ Esto significa que el sector agropecuario en general ha logrado aumentar su producción y su productividad sin sacrificar mano de obra, como ha ocurrido en otros países. Por otra parte, en algunos trabajos se sostiene que la productividad en las actividades agropecuarias de Nueva Zelanda ha mejorado notablemente como resultado de un uso más eficiente de los factores de producción, ya que el aumento en la productividad se ha convertido en un elemento esencial para el mejoramiento de la competitividad del sector agropecuario neozelandés..$^{28} \mathrm{En}$ este sentido, la productividad de la mano de obra y del capital en el sector agropecuario de Nueva Zelanda se triplicó entre 1975 y 1994, incluso muy por encima del incremento mostrado en la productividad

\section{Gráfica 4}

Nueva Zelanda: existencias de ganado ovino, 1975-1999

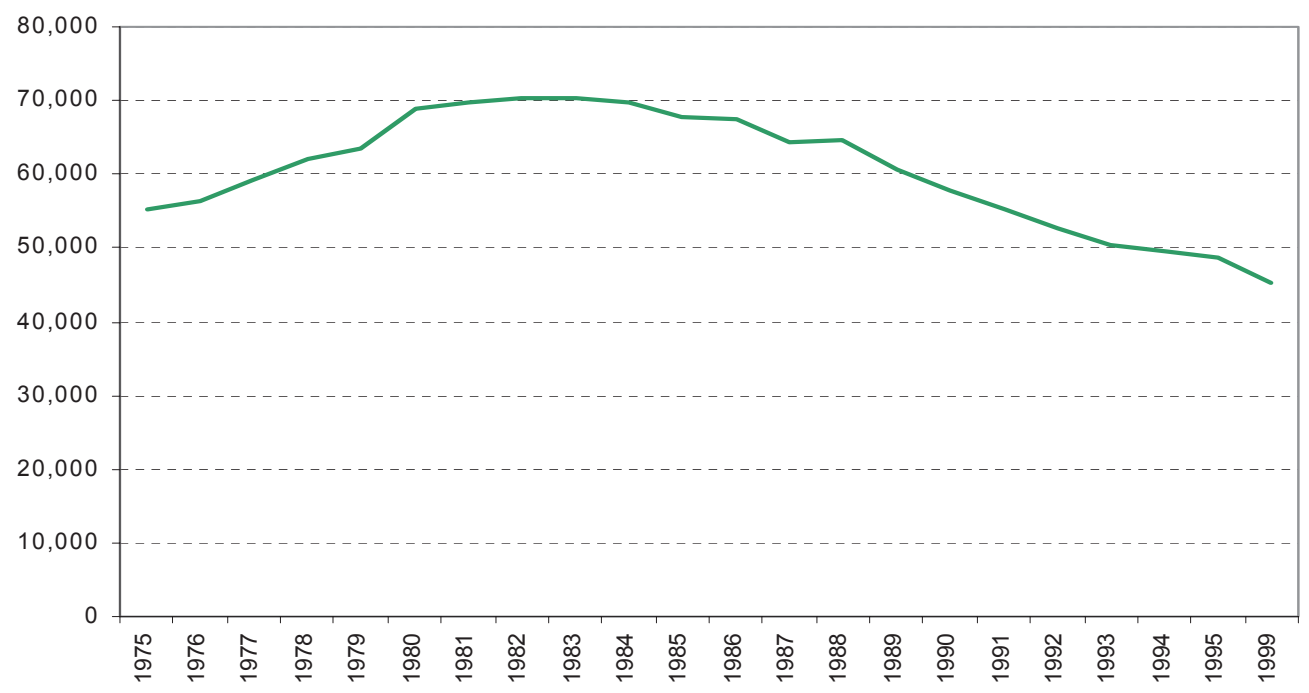

Fuente: elaborada con base en el Banco de datos de la FAO, Roma, 2001. 
del total de la economía en ese mismo periodo (cuadro 6).

Si bien la inversión en capital entre los productores aumentó sustancialmente entre 1991 y 1992, ya que se incrementó en 4.5 por ciento, en su mayor parte se destinó a la compra de tierras, más que a la adquisición de vehículos y maquinaria. Lo anterior significa que se desarrolló un mercado de la tierra, que necesariamente implicó un aumento en el valor de las unidades de producción rural. Para 1993, de acuerdo con algunos estudios, se estimó un crecimiento de 10 por ciento en la inversión en capital en el sector agropecuario de Nueva Zelanda. ${ }^{29}$

En el sector externo de Nueva Zelanda, además de continuar la tendencia a configurar una estructura de exportaciones agropecuarias más diversificada, se observó una lenta recuperación en el crecimiento de las mismas durante la primera mitad de los años noventa; entre 1990 y 1995 las exportaciones agropecuarias aumentaron en promedio 5 por ciento anual, en tanto que su participación en el total disminuyó de 54 a 45 por ciento. Sin embargo, en la segunda mitad volvieron a mostrar una pronunciada caída en concordancia con el menor crecimiento de la economía del país y como consecuencia del mayor grado de vulnerabilidad ante sucesos como la caída de los mercados de Asia. Para el periodo 1996-2000 los bienes agropecuarios exportados quedaron virtualmente estancados, al presentar un crecimiento de 0.06 por ciento promedio anual, mientras que su participación en el total aumentó de 47 a 50 por ciento, ante el mayor deterioro de otros sectores de exportación (gráfica 5).

En cuanto a las importaciones agropecuarias, éstas crecieron a un ritmo relativamente mayor que las exportaciones, al aumentar a un ritmo de casi 9 por ciento anual en la primera mitad de los noventa, pero en la segunda mitad también experimentaron un fuerte descenso al crecer a una tasa media menor a 3 por ciento anual, mientras que su participación en el total se mantuvo en alrededor de 8 por ciento durante toda la década de los noventa. Esto refleja la fuerte dependencia que aún mantiene la economía de Nueva Zelanda del sector agropecuario, pero también la rápida transformación del país a partir del mayor dinamismo de otros sectores productivos.

Dentro de las exportaciones agropecuarias, de las cuales 13 por ciento corresponden a productos agrícolas y 85 por ciento a productos pecuarios -incluidos otros bienes agroalimentarios como la leche

\section{Cuadro 6}

Nueva Zelanda: crecimiento de la productividad

(porcentaje anual)

\begin{tabular}{lrrrr}
\hline Concepto & \multicolumn{2}{c}{ Agricultura } & \multicolumn{2}{c}{ Total de la economía } \\
\hline & $1975-1985$ & $1985-1994$ & $1975-85$ & $1985-1994$ \\
\hline De la mano de obra & 2 & 6 & 1.1 & 2.7 \\
Del capital & 1.3 & 5.5 & -1.7 & -0.9 \\
De la razón capital/trabajo & 0.8 & 0.5 & 2.8 & 3.6 \\
Del total de los factores & 1.8 & 5.9 & 0.1 & 1.4 \\
\hline
\end{tabular}

Fuente: Allan Rae, "Agriculture can survive unilateral reforms: Lessons from down under", Conference on Food and Agricultural Policy Challenges for the Asia- Pacific, Manila, Filipinas, 1996. 


\section{Gráfica 5}

Nueva Zelanda: evolución del comercio agropecuaro, 1965-2001

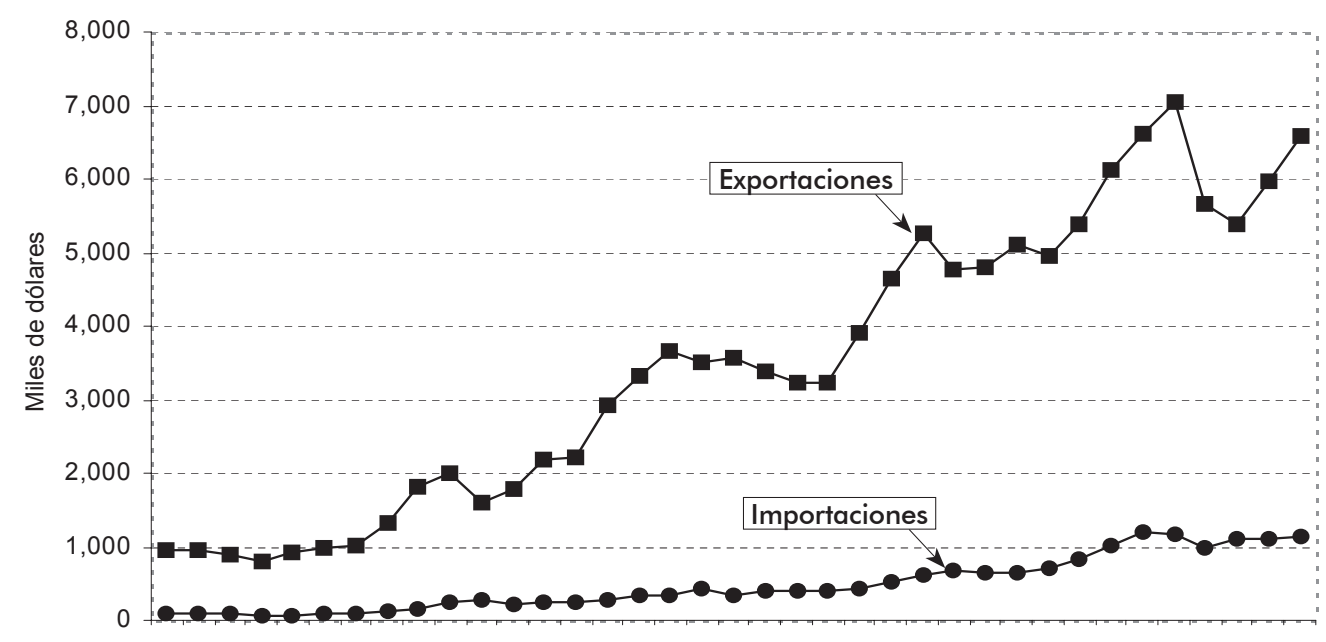

Fuente: elaborada con base en el Banco de datos de la FAO, Roma, 2001.

en polvo-, se puede apreciar un comportamiento distinto por tipos de productos durante la década de los años noventa. En un extremo están la lana, el kiwi y la carne, principalmente de ovino, que mostraron los más pobres desempeños al registrar la primera un crecimiento medio anual negativo de 8 por ciento, el segundo también un crecimiento negativo de 6 por ciento y la tercera con sólo 0.6 por ciento anual. Del lado opuesto están los productos lácteos como queso y mantequilla, que alcanzaron un crecimiento medio anual de casi 11 y 2 por ciento, respectivamente; y hortalizas y frutas, principalmente cebolla, calabaza y manzana, que crecieron en promedio $20,9 \mathrm{y}$ 15 por ciento, respectivamente (gráfica 6 ).

Es importante anotar que las anteriores modificaciones en el sector agropecuario se deben principalmente a los cambios en el contexto macroeconómico y microeconómico de Nueva Zelanda, pero también a eventos que tienen que ver con el mercado internacional. El problema radica en que, como Johnson reconoce, es muy difícil tratar de separar totalmente los efectos de uno y de otro, dada su estrecha interrelación. ${ }^{30}$ De lo que no hay duda, es que las políticas agrícolas implementadas en Nueva Zelanda a partir de 1984 han vuelto al sector agropecuario más sensible y más vulnerable a los cambios en las principales variables macroeconómicas, especialmente el tipo de cambio, pero al mismo tiempo lo han convertido en uno más eficiente y competitivo respecto al modelo anterior a la reforma económica.

\section{Adaptación de los agricultores de Nueva Zelanda}

Diversos estudios coinciden en que en Nueva Zelanda, particularmente durante las últimas dos décadas, las familias rurales han diversificado en forma creciente sus fuentes de ingreso a fin de mantener su ingreso familiar, así como de conservar sus granjas y la viabilidad de las mismas. ${ }^{31}$ 


\section{Gráfica 6}

Nueva Zelanda: principales productos exportados, 1965-1999

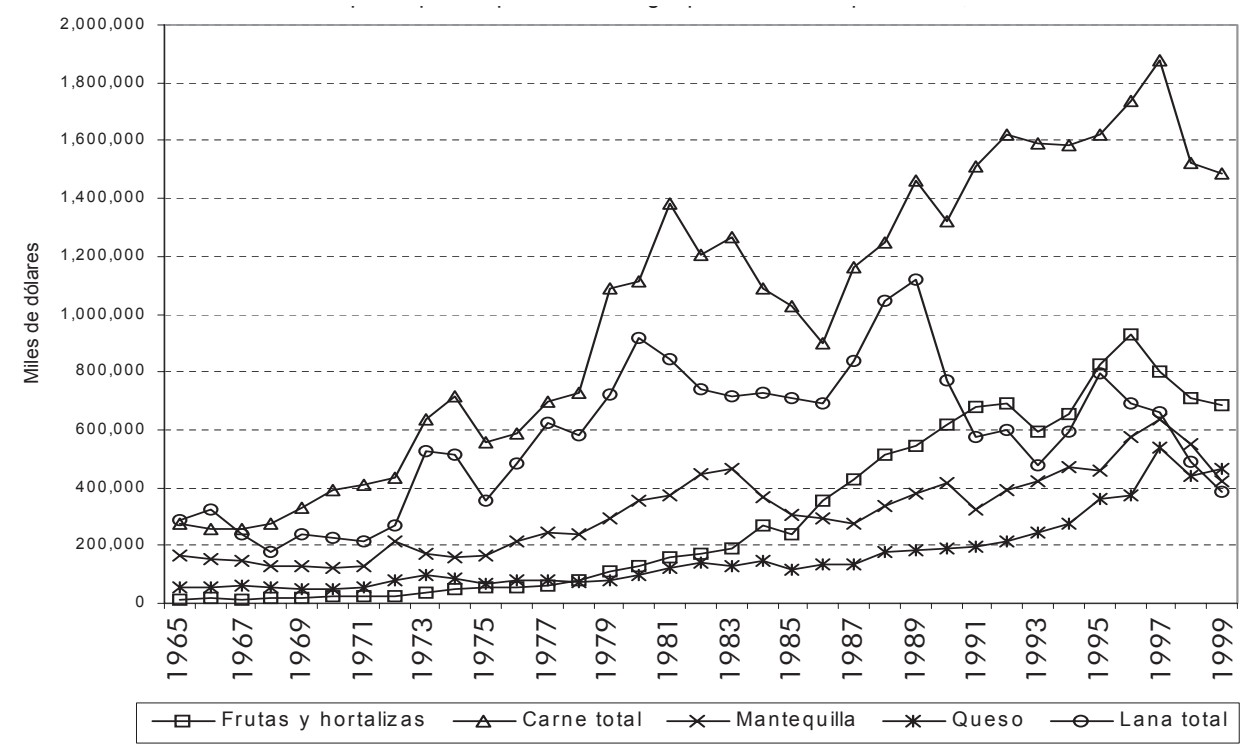

Fuente: elaborada con base en el Banco de datos de la FAO, Roma, 2001.

En ellos se destaca la tendencia cada vez mayor hacia la diversificación de las actividades productivas; por ejemplo, de la cría de ganado ovino y bovino a la cría de aves de corral, de venado y de avestruz; de la producción de leche fresca a la producción y comercialización de lácteos y leche en polvo; de la producción de cereales a la producción y procesamiento de frutas y hortalizas como kiwi, manzana, uva, pera, cebolla, calabaza y espárrago; de los viveros a la industria forestal, etc. Es decir, ha habido una progresiva diversificación en la estructura de producción hacia aquellos bienes de mayor valor agregado y que responden a las necesidades del mercado internacional, pero también a los requerimientos de una cada vez más grande y competitiva agroindustria trasnacional.

Lo anterior significa que la liberalización del sector trajo como consecuencia un mayor impulso a la forma en que los agricultores respondían a las señales de cambio que ya venían experimentando las actividades agropecuarias desde la década de los años setenta. Este proceso que, entre otros aspectos, tuvo que ver con cambios en el uso del suelo, en los tipos y niveles de producción, en el uso de insumos y en la inversión en capital, en respuesta a los cambios en los precios y costos de producción resultantes de las políticas neoliberales gubernamentales, se reflejó en nuevas tendencias en el ingreso agrícola, en el gasto y en la productividad. ${ }^{32}$ Por lo tanto, se puede establecer que las decisiones que están tomando los agricultores neozelandeses se han dado en estrecha relación con las decisiones del gobierno sobre la agricultura en el pasado, pero sobre todo con el proceso más reciente de cambio estructural de la agricultura.

De este forma, la agricultura neozelandesa se ha convertido en una industria dinámica y moderna en donde los productores han mostrado un alto grado de adaptabilidad a las nuevas condiciones de producción, gracias a que están apro- 
vechando mejor los factores que inciden en la producción, están utilizando más y mejores insumos, nuevas tecnologías y han sabido mantener su presencia en los mercados nacional e internacional. También esta transformación en la agricultura de Nueva Zelanda se ha caracterizado por un cambio trascendental en el papel del Estado, que abandonó casi totalmente su anterior función de agente regulador y gestor de la producción y comercialización agropecuaria, para convertirse actualmente en supervisor de una mejor operación de los mercados y de las condiciones ambientales y sanitarias en torno a la agricultura.

Otros factores igualmente importantes en esta transformación de la agricultura neozelandesa, han sido la mayor participación de la inversión privada en la agricultura y las mejores condiciones de financiamiento proporcionado por la banca privada, que ahora disfrutan los productores para la adquisición de insumos y maquinaria, lo cual ha permitido aumentos significativos en la productividad. En este sentido, en algunos trabajos se argumenta que el aumento en la productividad de la agricultura de Nueva Zelanda ha sido posible gracias a la combinación de un mejor uso de la tierra, nuevas tecnologías, mejores condiciones de financiamiento y otros apoyos, lo que ha permitido crear un modelo un tanto distinto al productivista que ha caracterizado la agricultura de algunos países como Estados Unidos, Canadá y otros de la Unión Europea. ${ }^{33}$

Este fenómeno tiene que ver precisamente con una progresiva articulación de la agricultura neozelandesa con la agroindustria global. Por un lado, a partir del crecimiento de la inversión privada en las actividades agrícolas, especialmente en la producción de frutas, hortalizas y lácteos y, por otro, a partir de la proliferación de nuevas relaciones contractuales entre los productores y las compañías agroindustriales. Incluso se afirma que ha habido una estrategia del gobierno para promover la venta de tierras a las compañías agroindustriales trasnacionales con objeto de orientar aún más la agricultura hacia el libre mercado y hacia su integración global con el mercado internacional.

En este proceso, en donde los agricultores son cada vez menos independientes, las relaciones de producción están cambiando rápidamente. Ahora el control de la producción se está trasladando fuera de las granjas de producción, de manera que los bancos y las compañías agroindustriales han adquirido un gran poder de decisión en los procesos de producción, por un lado, y las transnacionales han desarrollado una gran capacidad y nuevos mecanismos para apropiarse de las ganancias mediante nuevas formas de vinculación con los agricultores, por otro. De ahí que los intentos de Nueva Zelanda en el pasado, tendientes a buscar una mayor diversificación económica y comercial, 
están siendo reemplazados por una nueva forma de dependencia de sus agricultores, que ha implicado la concentración de la producción, transformación y comercialización de los bienes agropecuarios en manos de unas cuantas compañías agroindustriales nacionales y trasnacionales. ${ }^{34}$

Por otra parte, en estrecha relación con la diversificación de las actividades económicas en el medio rural, y en cierta forma como una respuesta a la progresiva marginalización de algunos agricultores, existe una fuerte tendencia entre la población rural hacia el empleo en actividades no agrícolas y otras actividades empresariales alternativas dentro de sus propias granjas. Cabe señalar que algunas de éstas son nuevas actividades con una creciente demanda en el país, que han venido a constituirse en actividades complementarias con las existentes, o bien en nuevas formas de empleo que han implicado modificar total o en gran parte el uso de la tierra, incluso de aquéllas con poca o ninguna conexión con las actividades agrícolas. Entre estas actividades están el turismo rural, los viveros hortícolas especializados, el procesamiento de productos agrícolas y pecuarios, los servicios y consultoría agrícola, los servicios generales, la manufactura ligera y las artesanías. ${ }^{35}$

Esto muestra la amplitud e importancia de la pluriactividad que están desarrollando las explotaciones rurales en Nueva Zelanda, en donde está cobrando cada vez más fuerza el empleo en actividades no agrícolas y en actividades alternativas dentro de la misma granja. Es importante tomar en consideración que este proceso de adaptación de los agricultores y de la diversificación del ingreso se ha dado en función de su disponibilidad de recursos naturales, económicos y humanos, pero también de diversos factores externos tales como el acceso y comportamiento de los mercados externos, los precios de las mercancías, los costos del capital y el mercado de trabajo, entre otros factores.

\section{Conclusiones}

Como parte de las reformas económicas implementadas en Nueva Zelanda en 1984, impulsadas para restaurar la competitividad de la economía y corregir los desequilibrios macroeconómicos en que había caído el país, en el sector agropecuario se llevó a cabo un amplio proceso de reestructuración que incluyó la eliminación de los subsidios a los insumos para la agricultura, la desaparición de los controles sobre los precios agrícolas, la eliminación de las concesiones fiscales y de los subsidios a las tasas de interés y al crédito que habían disfrutado los productores. En términos más amplios, se puede establecer que las reformas económicas en general y de la agricultura en particular, han tenido un impacto positivo, no obstante que el proceso de ajuste de los agricultores ha sido difícil y requirió más tiempo del esperado. El éxito de las reformas se ha reflejado en las mejores condiciones del nuevo ambiente desregulado en que ahora se desenvuelven los agricultores.

Cabe subrayar que las reformas introducidas en el sector vinieron a consolidar importantes cambios que ya se venían dando en la estructura de producción. Al mismo tiempo que hubo una diversificación en la producción, aumentó notablemente la productividad agrícola, debido en gran parte a que los agricultores están usando sus recursos con mayor eficiencia. $\mathrm{Al}$ interior del sector se observa que se han liberado recursos que estaban subutilizados hacia otras actividades con mayor valor agregado, lo cual ha propiciado que la agricultura haya recuperado su dinamismo y su importancia dentro de la economía de Nueva 
Zelanda. Como consecuencia del proceso de ajuste, sobre todo en el corto y mediano plazo, se produjo una significativa elevación de los costos de producción, lo que inevitablemente repercutió en la rentabilidad de las unidades de producción rural. Y, aun cuando no formaba parte de la estrategia de la reforma, el gobierno tuvo que llevar a cabo una serie de medidas de apoyo al productor para tratar de aminorar los efectos del ajuste sobre la agricultura.

El inestable comportamiento de la producción agropecuaria en los primeros años de la reforma, se explica por la combinación de diversos factores tanto internos como externos; entre ellos: los desequilibrios producto de las mismas reformas, la severidad del ajuste producto del drástico recorte de los subsidios y las distorsiones comerciales en los mercados agrícolas internacionales derivadas de los esquemas proteccionistas de otros países. A partir de 1992, de manera paralela a la economía, la producción agropecuaria, especialmente frutas, hortalizas y lácteos, comenzaron una etapa de rápido crecimiento al mejorar la eficiencia en el uso de los recursos por parte de los productores y de un mejor posicionamiento de estos productos en los mercados nacional e internacional. Un elemento esencial en este proceso fue la creciente vinculación de la agricultura neozelandesa con la agroindustria nacional y trasnacional, así como capacidad de respuesta de los productores para ajustarse a las nuevas condiciones de producción.

Actualmente la agricultura de Nueva Zelanda se ha convertido en una industria dinámica orientada hacia el exterior, los exportadores se han adaptado ya a las nuevas condiciones del mercado y están respondiendo favorablemente al nuevo ambiente desregulado, con pocas barreras a las importaciones y una mínima inter- vención del gobierno. De igual forma, la estructura de comercialización interna ha sido modificada sustancialmente, ya que las actividades de las juntas de productores relacionadas con la comercialización en el mercado interno han sido limitadas y suspendidas algunas de sus operaciones de regulación del mercado. Los agricultores han respondido a las nuevas oportunidades del mercado modificando el uso de la tierra, utilizando nuevos insumos y tecnologías, subdividiendo o aumentando la escala de producción y mediante nuevos esquemas de asociación. Es decir, han respondido a las nuevas señales del mercado con una mayor diversificación de la producción, pero también de sus fuentes de ingreso, de acuerdo con sus capacidades y disponibilidad de recursos.

Sin embargo, todo indica que el control de los procesos de producción se ha estado moviendo de los productores hacia las compañías agroindustriales, las cuales están adquiriendo cada vez más una mayor importancia y un gran poder de decisión sobre la agricultura mediante la inversión en los procesos de producción, distribución y comercialización de la producción agropecuaria. Esto es, la agricultura neozelandesa está pasando a un esquema eminentemente capitalista, caracterizado por procesos de producción y distribución de alimentos vertical y horizontalmente integrados, en donde los agricultores, especialmente los más pequeños, están recurriendo cada vez más a actividades complementarias y a otras fuentes de ingreso no agrícola. ny

\section{Notas}

1 Ministry of Agriculture and Forestry, 2001.

2 Ídem.

3 FMI, 2005.

4 Far Eastern Economic Review, 2000; ocDE,1996, p. 194.

5 Ídem. 
6 Véase Jacobsen, Scobie y Duncan, 1995.

7 Véase Sandrey y Scobie, 1994, p. 1041.

8 OCDE, 1994, pp. 71-72.

9 Ídem.

10 Ibid., p. 73.

11 Sandrey y Scobie, op. cit., 1994.

12 Idem.

13 OCDE, op. cit., 1996.

14 Véase Johnson, Schroeder y Taylor, 1989.

15 Ibid., p. 141.

16 Para un análisis de las reformas económicas se sugiere ver Evans, Grimes y Wilkinson, 1996.

17 Véase Johnston y Frengley, 1994, p. 1034.

18 Véase Rae, 1996.

19 Ibid., p. 12.

20 Johnston y Frengley, op. cit., 1994, p. 1035.

21 OCDE, op. cit., 1994, p. 143.

22 Idem.

23 Idem.

24 Ibid., pp. 156-157.

25 Véase Johnson, 1993, p. 88.

26 Idem.

27 Ministry of Agriculture and Forestry, op. cit., 2001.

28 Véanse Johnson, op. cit., 1993, pp. 390-391; Rae, op. cit., 1996, pp. 9-10.

29 Idem.

30 Véase Johnson, 1991, pp. 130-131.

31 Entre estos estudios destaca el de Taylor y McCrostie, 1997.

32 Véase Sandrey y Reynolds, 1990, pp. 156-182.

33 Véase Lawrence, 1999, p. 46.

34 Véase Burch, Rickson y Lawrence, 1999.

35 Taylor y McCrostie, op. cit., 1997.

\section{Referencias bibliográficas}

Burch, David, Roy E. Rickson, y Geoffrey Lawrence (1999) Globalization and Agri-Food Restructuring: Perspectives from the Australasia Region. Inglaterra: Ashgate Publishing Limited.

CEPAL (1993) El proceso exportador en tres agriculturas templadas del Hemisferio Sur (Australia, Nueva Zelanda y Sudáfrica), Unidad de Desarrollo Agrícola de la División de Desarrollo Productivo y Empresarial, documento LC/R.1350, 30 de diciembre, Santiago de Chile.

Evans, Lewis, Arthur Grimes, y Bryce Wilkinson (1996) "Economic Reform in New Zealand 1984-1995: The Pursuit of Efficiency", Journal of Economic Literature, vol. xxxIv, diciembre.

FaO (2001) Banco de datos, Roma.

Far Eastern Economic Review (2000), abril 13.

Fondo Monetario Internacional (FMI) (1996) World Economic Outlook, octubre, Washington, D. C. (2003) Estadísticas financieras internacionales, 1992, 1995, 1998 Y 2003, Washington, D. C.
Gardner, Bruce L. (1994) "Liberalization in New Zealand Agriculture. Discussion", American Journal of Agricultural Economics, vol. 76, núm. 5, diciembre, American Agricultural Economics Association.

Jacobsen, Veronica, Grant M. Scobie, y Alex Duncan (1995) "Statutory Intervention in Agricultural Marketing. A New Zealand Perspective", World Bank Technical Paper, núm. 283, Washington, D. C.

Johnson, R. W. M. (1991) "Current Changes in New Zealand Agriculture: A Review", Review of Marketing and Agricultural Economics, vol. 59, núm. 2, agosto, Estados Unidos.

_ (1993) "New Zealand Agricultural Policy Review: 1991-1993", Review of Marketing and Agricultural Economics, vol. 61, núm. 3, diciembre, Estados Unidos.

Johnson, R. W. M., W. R. Schroeder, y N. W. Taylor (1989) "Deregulation and the New Zealand Agricultural Sector: A Review", Review of Marketing and Agricultural Economics, vol. 57, núm. 1, 2, 3, Estados Unidos.

Johnston, Warren E., y Gerard A. G. Frengley (1994) "Economic Adjustments and Changes in Financial Viability of the Farming Sector: The New Zealand Experience", American Journal of Agricultural Economics, vol. 76, diciembre, Estados Unidos.

Lawrence, Geoffrey (1999) "Contemporary agri-food restructuring: Australia and New Zealand", Globalization and Agri-Food Restructuring. Perspectives from the Australasia Region. Inglaterra: Ashgate Publishing Limited.

Ministry of Agriculture and Forestry (2001) New Zealand: a Land of Contrasts, Nueva Zelanda. http:// www.maf.govt.nz/MAFnet.

OCDE (1994) Farm Employment and Economic Adjustment in OECD Countries, Francia.

- (1996) OECD Economic Surveys. New Zealand, Francia.

__ (1996, 1999, 2001) Agricultural Policies, Markets and Trade in OECD Countries, Monitoring and Evaluation, Francia.

Rae, Allan (1996) Agricultural can Survive Unilateral Reforms: Lessons from Down Under, Conference on Food and Agricultural Policy Challengers for the Asia-Pacific, Manila, Filipinas. 1-3 de octubre.

Sandrey, Ron A., y Grant M. Scobie (1994) "Changing International Competitiveness and Trade: Recent Experience in New Zealand Agriculture", American Journal of Agricultural Economics, vol. 76, núm. 5, diciembre. Estados Unidos: American Economic Association.

Sandrey, Ron, y Russell Reynolds (1990) Farming without Subsidies. New Zealand's Recent Experience. Nueva Zelanda: Ministry of Agriculture and Fisheries.

Taylor, Nick, y Heather McCrostie (1997) "Entrepreneurship in New Zealand Farming: A Study of Alternative Enterprises on Farms", MAF Policy Technical Paper, junio, Nueva Zelanda. 